\title{
ESTRUTURA DA COMUNIDADE DE ARTRÓPODES DE SOLO EM DIFERENTES FITOFISIONOMIAS DA RESERVA PARTICULAR DO PATRIMÔNIO NATURAL - SESC PANTANAL, BRASIL
}

\author{
J. R. MUDREK ${ }^{1}$ e E. V. MASSOLI JUNIOR ${ }^{2}$ \\ Centro Universitário de Várzea Grande (UNIVAG) \\ jessicamudrek@hotmail.com¹; massolijr@hotmail.com²
}

Artigo submetido em setembro/2013 e aceito em fevereiro/2014

\section{RESUMO}

As teias alimentares presentes na serrapilheira são de extrema importância para que haja a ciclagem dos nutrientes presentes no solo. A fauna de artrópodes de solo vem sendo estudada no Pantanal matogrossense para que ocorra identificação dos grupos, bem como sua riqueza e distribuição. O objetivo deste trabalho foi analisar a comunidade de artrópodes em duas fitofisionomias do Pantanal, além de verificar a relação da abundância e/ou riqueza da fauna com a altura da serrapilheira. O estudo foi realizado em Abril de 2012, na RPPN Sesc Pantanal, no município de Poconé, Mato Grosso, Brasil. Foram instaladas dez armadilhas de queda (pitfalls) dentro de duas parcelas de 50x50 m, com fitofisionomias distintas, Cerrado sensu stricto e
Floresta semidecidual. A serrapilheira foi mensurada por meio de fita métrica (raio de $30 \mathrm{~cm}$ ). O material foi encaminhado ao laboratório de zoologia, triado e identificado. Para realização da análise de variância e correlação dos artrópodes com serrapilheira foi utilizado o programa Systat 10 . Houve diferença significativa na riqueza entre as duas fitofisionomias, por sua vez para a abundância não foi significativa. Não houve correlação entre a altura de serrapilheira e a riqueza/abundância de artrópodes. As ordens mais abundantes corroboram com outros trabalhados, porém seriam necessárias amostragens mais longas para uma lista mais completa das ordens identificadas.

PALAVRAS-CHAVE: Invertebrados, Fauna edáfica, Pantanal de Poconé, Cerrado, Floresta Semidecidual, Serrapilheira.

\section{STRUCTURE OF ARTHROPOD COMMUNITY SOLO IN DIFFERENT VEGETATION TYPES OF NATURAL RESERVE OF PRIVATE EQUITY - SESC PANTANAL, BRAZIL}

\begin{abstract}
The food webs that exist in litter are extremely important for the nutrient cycling in the soil to happen. The arthropod fauna of the soil has been studied in the Pantanal region in order to identify the groups as well as their wealth and distribution. The objective of this study was to analyze the community of arthropods in two vegetation types of the Pantanal, and verify the relation between abundance and/or richness of the fauna with the height of the litter. The study was conducted in April 2012, the PRNP SESC Pantanal, municipality of Poconé, Mato Grosso, Brazil. Ten pitfall traps were installed in two plots of $50 \times 50 \mathrm{~m}$, with distinct vegetation types, cerrado stricto sensu and semi-deciduous forest. The
\end{abstract}

litter was measured by tape measure (radius of $30 \mathrm{~cm}$ ). The material was sent to the zoology laboratory, screened and identified. To perform the analysis of variance and correlation of litter arthropods the Systat 10 software was used. There was a significant difference in wealth between the two forest types, but to the abundance it was not significant. There was no correlation between the height of litter and richness/abundance of arthropods. The most abundant orders corroborate other studies, but would necessary a long sampling for a more complete list of identified orders.

KEYWORDS: Invertebrates, Soil Fauna, Pantanal of Poconé, Cerrado, Semidecidual Forest, Litter. 


\section{INTRODUÇÃO}

No Pantanal Mato-grossense a fauna de solo vem sendo estudada para que haja identificação dos grupos de artrópodes, bem como sua riqueza e distribuição (MARQUES et al., 2010). As comunidades de artrópodes participam ativamente nas teias alimentares e influenciam o processo de decomposição dos detritos podendo altera o funcionamento do ecossistema (FERREIRA; MARQUES, 1998).

O Pantanal é uma extensa planície de inundação e suas variações de topografia e solo fazem com que áreas alaguem periodicamente (SIGNOR et al., 2010), e que algumas permaneçam sempre secas, esse alagamento é ocasionado pela pluviosidade local e pelo transbordamento de rios (JUNK et al., 1989).

A vegetação do Pantanal é caracterizada por diversos fatores, como o tipo de solo, duração de inundação, stress pelo fogo, intensidade de pastejo pelo gado e a limpeza das áreas para pecuária (SIGNOR et al., 2010). A ocorrência de cada fitofisionomia é o resultado da combinação destes fatores (FANTIN-CRUZ et al., 2010), deste modo, o Pantanal pode apresentar as seguintes fitofisionomias: floresta semidecidual, cerrado, cerradão, cerrado aberto, campo de murundu, paratudal, piuval, cordilheira, cambarazal e campo inundável (NUNES DA CUNHA; JUNK, 2011).

No Pantanal, o Cerrado é caracterizado por arbustos e subarbustos, árvores baixas, inclinadas, com ramificações irregulares e retorcidas (RIBEIRO; WALTER, 1998) conhecido especificamente como Cerrado sensu stricto. Dentro desta fitofisionomia existe uma grande diversidade de habitats e alternância de espécies, apesar de apresentar um solo pobre em nutrientes (KLINK; MACHADO, 2005).

As florestas estacionais semidecíduas, ocorrem em regiões de solo elevado dentro do Pantanal e possuem solos relativamente ricos em nutrientes, desenvolvidos a partir de rochas básicas. Estas matas são também denominadas florestas estacionais por perderem as folhas na estação seca (FELFILI et al., 2005).

Dentro das fitofisionomias, uma das principais fontes de nutrientes é proveniente da serrapilheira (XIONG; NILSSON, 1999), composta pelas folhas, frutos e galhos senescentes e, em menor proporção por restos de animais e material fecal (MARQUES et al., 2010). As teias alimentares presentes são de extrema importância para que haja a ciclagem dos nutrientes (BATTIROLA et al., 2007), sendo a principal fonte de energia para a base da cadeia alimentar, composta principalmente por artrópodes (CORREIA; OLIVEIRA, 2000), que são responsáveis pela fragmentação da matéria orgânica, acelerando o processo de decomposição.

A composição de espécies e a estrutura da comunidade de serrapilheira são dependentes de vários fatores: tipo de formação vegetal, solo, climatologia local e diversidade de microhabitats. A quantidade de recursos e a variedade ambiental são importantes na determinação da diversidade (RIBAS et al., 2003). As diferentes características ambientais das fitofisionomias podem compor distintas riquezas e abundâncias de artrópodes de solo. 
Neste sentido, o objetivo do trabalho foi analisar a comunidade de artrópodes de duas fitofisionomias do pantanal, além de verificar a relação da abundância e/ou riqueza dessa fauna com a altura da serrapilheira.

\section{MATERIAL E MÉTODOS}

\section{1 Área de estudo}

O estudo foi realizado em Abril de 2012, no Parque Baia de Pedras, na Reserva Particular do Patrimônio Natural (RPPN) Sesc Pantanal, no município de Poconé, Mato Grosso (Figura 1). Essa unidade de conservação possui 4.500 hectares, apresentando grande diversidade de paisagens do Pantanal, dentre elas, Cerrado sensu stricto e Floresta semidecidual, as quais foram utilizadas no estudo.

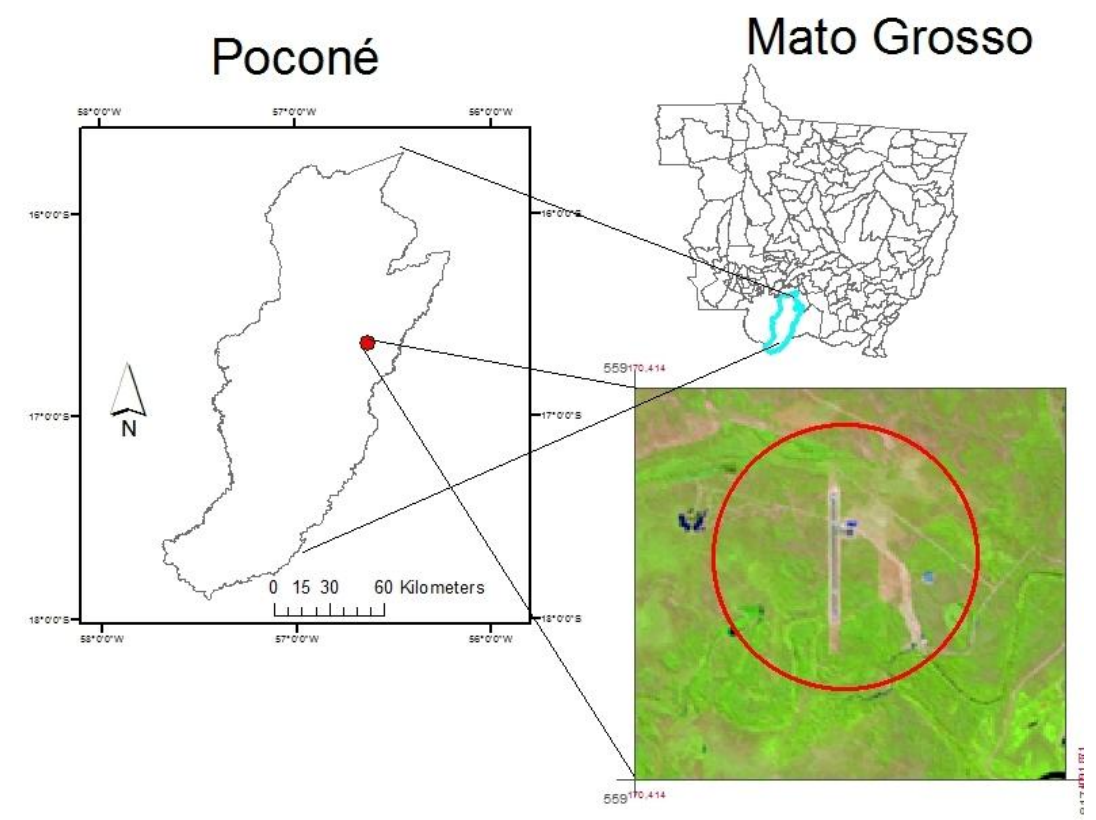

Figura 1 - Área de estudo. Parque Baia de Pedras, na RPPN do Sesc Pantanal, município de Poconé, Mato Grosso, Brasil.

A temperatura média anual do Pantanal é de 25으, exceto nos meses de setembro a dezembro que as temperaturas alcançam 40C (SIGNOR et al., 2010), possui duas estações bem definidas: a estação seca, de maio a setembro e, a estação chuvosa, de outubro a abril, a precipitação anual média dos últimos 14 anos foi de $1220 \mathrm{~mm}$, variando de $239 \mathrm{~mm}$ na estação seca a 1002 mm na estação chuvosa (VIANA; ALVALÁ, 2011).

\subsection{Coleta de dados}

Duas parcelas de $50 \times 50 \mathrm{~m}$ foram instaladas. Em cada parcela, 10 armadilhas de queda (pitfalls) foram distribuídas aleatoriamente. Os pitfalls consistem em garrafas pets de dois litros, cortadas ao meio e enterradas ao nível do solo, onde foram acrescentados $200 \mathrm{~mL}$ de álcool $70 \%$, e duas gotas de detergente (para quebrar a tensão superficial do álcool), com o intuito de matar e conservar os artrópodes capturados. A serrapilheira foi medida com auxílio de trena métrica em três pontos próximos ao pitfalls, dentro de um raio de $30 \mathrm{~cm}$ do entorno. 
O estudo totalizou 24 horas de esforço amostral por pitfall. O material foi transferido para potes de plástico com álcool 70\%, identificados previamente, levados ao laboratório de Zoologia do Centro Universitário de Várzea Grande (UNIVAG), e triados em lupa (aumento de 50x).

Os artrópodes foram identificados à nível de ordem, com auxílio de bibliografia especializada (BRUSCA; BRUSCA, 2007), colocados em placa de Petri, quantificados e armazenados em tubos criogênicos com álcool $70 \%$.

Realizamos a Análise de Variância (ANOVA) para testar se houve diferença significativa entre a abundância e riqueza nas diferentes fitofisionomias (Cerrado e Floresta semidecidual); e correlação de Pearson como índice de associação, para verificar se houve correlação entre a altura da serrapilheira com a riqueza/abundância de artrópodes. Para essas análises utilizamos o software estatístico Systat 10.

\section{RESULTADOS}

A comunidade de artrópodes de solo na área do Parque da Baia de Pedras foi constituída por grande diversidade de grupos, sendo coletados 1.422 indivíduos pertencentes a 13 Ordens, distribuídas em quatro Classes, nas duas fitofisionomias amostradas (Tabela 1).

As ordens mais abundantes foram: Hymenoptera com 29,5\% ( $N=420)$, Collembola com $22,2 \%(\mathrm{~N}=315)$ e Coleoptera com $15,1 \%(\mathrm{~N}=215)$, as outras 10 ordens apresentaram 33,2\% $(\mathrm{N}=472)$ do total observado.

Tabela 1 - Estrutura da comunidade de artrópodes nas diferentes fisionomias amostradas, em Abril de 2012, na Baia de Pedras, RPPN SESC Pantanal, Poconé, Mato Grosso, Brasil.

\begin{tabular}{clcc}
\hline & TAXA & \multicolumn{2}{c}{ FITOFISIONOMIA } \\
\hline Classe & \multicolumn{1}{c}{ Ordem } & Cerrado & Floresta Semidecidual \\
\hline Insecta & Hymenoptera & 289 & 131 \\
& Coleoptera & 98 & 117 \\
& Orthoptera & 51 & 20 \\
& Dermaptera & 39 & 124 \\
& Hemiptera & 33 & 34 \\
& Blattodea & 2 & 8 \\
& Diptera & 0 & 55 \\
Arachnida & Acarina & 22 & 33 \\
& Aranae & 15 & 19 \\
& Pseudoscorpiones & 8 & 4 \\
Malacostraca & Decapoda & 2 & 0 \\
& Isopoda & 0 & 39 \\
\hline \multirow{5}{*}{ Entognatha } & Collembola & 226 & 637 \\
\hline & Total & 785 & 1422 \\
& Total Geral & - & \\
& & & 39 \\
\hline
\end{tabular}

Comparando a riqueza e abundância entre as fitofisionomias, observa-se que no Cerrado, as ordens mais abundantes foram: Hymenoptera (36,8\%), Collembola $(28,8 \%)$ e Coleoptera 
$(12,5 \%)$, Decapoda esteve presente apenas nesta fisionomia. Dentro da Floresta semidecidual, as ordens mais abundantes foram: Hymenoptera (20,6\%), Dermaptera (19,5\%) e Coleoptera $(18,4 \%)$, sendo que Isopoda e Diptera foram registradas apenas nesta fisionomia (Figura 2$).$

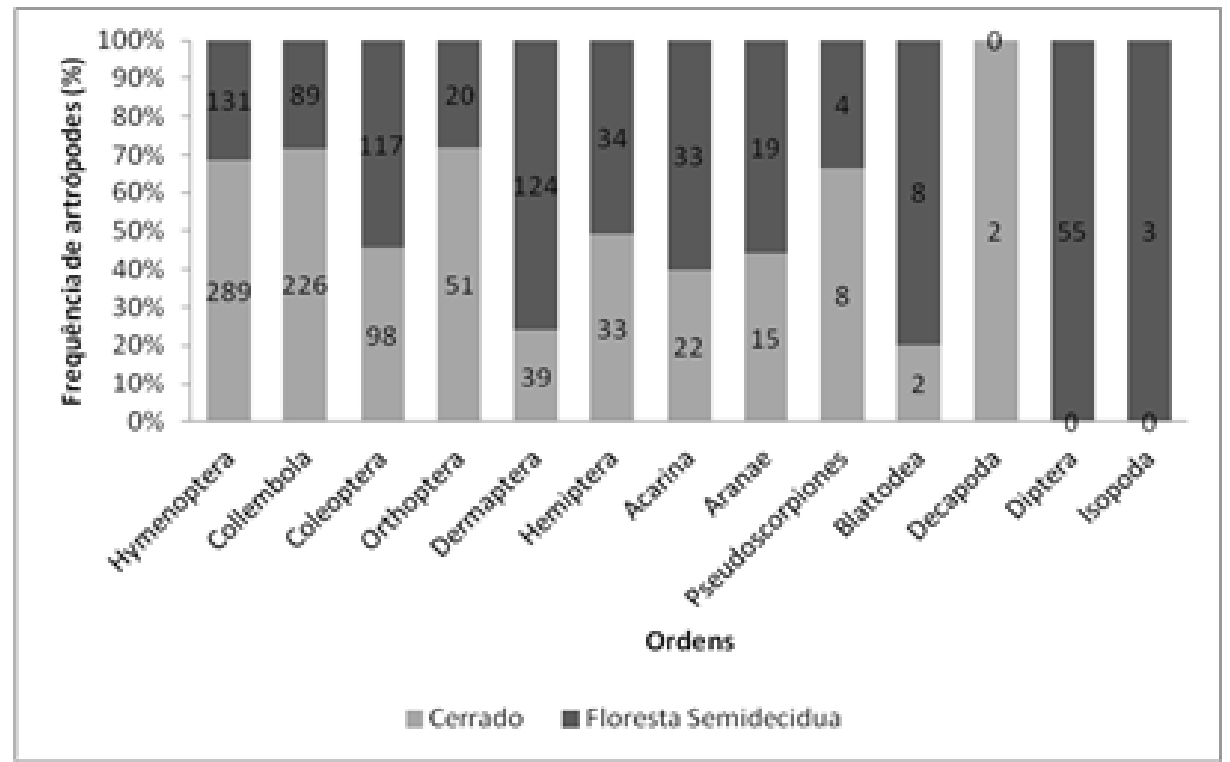

Figura 2 - Frequência relativa de artrópodes nas diferentes fitofisionomias (Cerrado e Floresta semidecidual).

Pela Análise de Variância (ANOVA) verificou-se que há diferença significativa $(p=0,018)$ na riqueza de artrópodes entre as amostras de Cerrado e Floresta semidecidual, com alto valor de Fratio $(6,76)$, havendo pouca diferença na riqueza entre as amostras de mesma fisionomia (Figura $3)$. Enquanto que para a abundância, observa-se que não há diferença significativa $(p=0,320)$ entre as amostras de Cerrado e Floresta semidecidual, com baixo valor de F-ratio $(1,045)$, ocorrendo muita variação na abundância entre as amostras de mesma fisionomia (Figura 4).

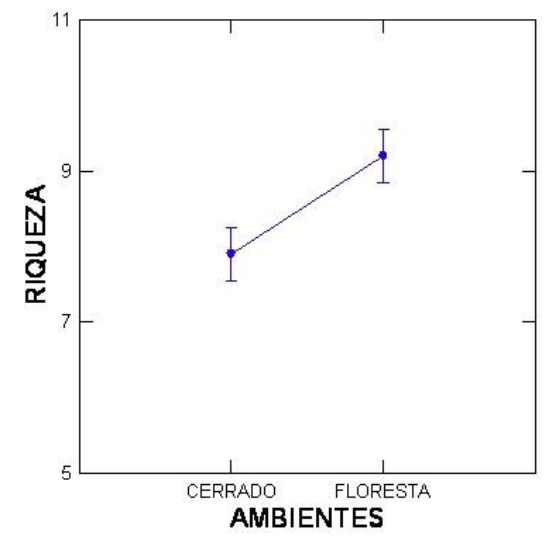

Figura 3 - Análise de Variância (ANOVA) da Riqueza de artrópodes entre o Cerrado e a Floresta semidecidual.

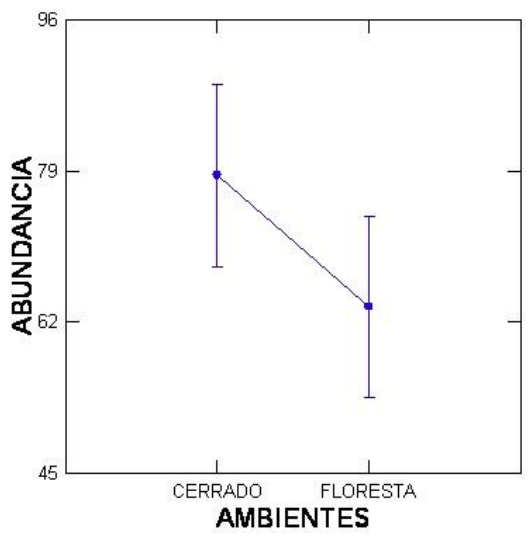

Figura 4 - Análise de Variância (ANOVA) da Abundância de artrópodes entre o Cerrado e a Floresta semidecidual.

$\mathrm{Na}$ análise de correlação constata-se que ambos os atributos da comunidade de artrópodes considerados, riqueza $(R=0,246)$ e abundância $(R=-0,145)$, não apresentaram correlação com a altura da serrapilheira. 


\section{DISCUSSÃO}

Os resultados obtidos no presente estudo, referentes às ordens mais abundantes corroboram com os obtidos por Marques et al. (2011), identificando as mesmas (Hymenoptera, Coleoptera e Collembola) em um estudo realizado no Pantanal do Pirizal, município de Nossa Senhora do Livramento, Mato Grosso. Praxedes et al. (2009), obteve resultados semelhantes, na Floresta Nacional de Caxiuanã, município de Melgaço, Pará.

A grande representatividade da ordem Hymenoptera pode ser atribuída aos diferentes papeis ecológicos desempenhados nos ecossistemas, atuando como polinizadores, dispersores de sementes, predadores, parasitoides, ou se alimentando de tecidos vegetais, de fungos ou de outros artrópodes (BATTIROLA et al., 2007; FEITOSA et al., 2007), assim como são considerados insetos predominantes na maioria dos ecossistemas terrestres (ALONSO; AGOSTI, 2000).

Coleoptera e Collembola, comumente estão associados à matéria orgânica, nas quais desempenham muitas vezes, papel de decompositores da matéria dependendo do substrato para sua sobrevivência (BATTIROLA et al., 2007). A ordem Collembola habita, de preferência lugares úmidos, no solo humoso, em folhas caídas, bainhas de folhas de plantas, entre musgos e detritos vegetais em decomposição (BUZZI, 2002).

Battirola et al. (2007), em estudo realizado em Pirizal, município de Nossa Senhora do Livramento, Mato Grosso, associaram os artrópodes à copa de uma palmeira (Attalea phalerata), obtendo resultados semelhantes aos do presente estudo, onde Coleoptera foi a ordem mais abundante seguido por Psocoptera.

A presença de Diptera apenas na floresta semidecidual pode estar relacionada com o desenvolvimento larval de alguns gêneros, que ocorrem em solo úmido, ou em detritos ricos em matéria orgânica em decomposição nos ambientes florestais, além disso, esses animais utilizam o folhiço como abrigo ou para forragear (BATTIROLA et al., 2007; ZARDO et al., 2010).

No estudo realizado na Estação Ecológica Serra das Araras, no município de Porto Estrela, Mato Grosso, Zardo et al. (2010), compararam a fauna edáfica do Cerrado e da Mata de Galeria, os autores verificaram que o cerrado foi mais rico e abundante em comparação à mata de galeria, o que corrobora com parte dos resultados do presente estudo, em que o cerrado se mostrou mais abundante.

De acordo com Ferreira e Marques (1998), a maior riqueza na floresta semidecidual está relacionada com a maior complexidade ambiental, importante para a existência da maior diversidade, em decorrência das condições mais favoráveis, como abrigo, local para forrageamento e reprodução. Os mesmos autores realizando um estudo comparativo entre eucaliptal e mata, verificaram uma maior diversidade na mata, isso pode ser atribuído a maior qualidade nutritiva da serrapilheira quando comparada ao eucaliptal.

Esses resultados contribuem para o conhecimento da biodiversidade de Artrópodes do Pantanal e podem ser aplicados em avaliações de impactos ambientais e planos de manejo de unidades de conservação. 


\section{CONCLUSÕES}

As ordens Hymenoptera, Collembola e Coleoptera são mais abundantes nas fisionomias estudadas. A floresta semidecidual apresentou maior riqueza, enquanto a maior abundância foi observada no cerrado, isso se deve às características estruturais de cada ambiente.

\section{AGRADECIMENTOS}

Ao Ms. Luis Antônio Solino de Carvalho pelo valioso auxílio nas atividades desenvolvidas neste trabalho, à Neidevon Realino de Jesus pelo auxilio em campo e à técnica do Laboratório de Zoologia do UNIVAG, Paola Scame, pela triagem do material. Ao Governo Federal pela bolsa concedida (ProUni) ao primeiro autor. Esse trabalho teve apoio do UNIVAG (Programa de Iniciação Científica 2011/2).

\section{REFERÊNCIAS BIBLIOGRÁFICAS}

1. ALONSO, L. E.; AGOSTI, D. Biodiversity studies, monitoring, and ants: an overview. In: AGOSTI, D.; MAJER, J. D.; ALONSO, L. E.; SCHULTZ, T. R. Standard methods for measuring and monitoring biodiversity. Smithsonian Institution: Washington, p. 1-8, 2000,

2. BATTIROLA, L. D.; ADIS, J.; MARQUES, M. I.; SILVA, F. H. O. Comunidade de Artrópodes Associada à Copa de Attalea phalerata Mart. (Arecaceae) durante o Período de Cheia no Pantanal de Poconé, MT. Neotropical Entomology, n. 36, v. 5, p. 640-651, 2007.

3. BRUSCA, R. C.; BRUSCA, G. J. Invertebrados. ed 2. Guanabara Koogan, Rio de Janeiro, 2007, $968 \mathrm{p}$.

4. BUZZI. Z. J. Ordem Collembola, Cap. 4. Entomologia Didática. Paraná, Editora UFPR, 2002, $343 \mathrm{p}$.

5. CORREIA, M. E. F.; OLIVEIRA, L.C.M. de. Fauna de Solo: Aspectos Gerais e Metodológicos. Seropédica: Embrapa Agrobiologia, 2000, 46 p.

6. FANTIN-CRUZ, I.; GIRARD, P.; ZEILHOFER, P.; COLLISCHONN, W.; NUNES DA CUNHA, C. Unidades fitofisionômicas em mesoescala no Pantanal Norte e suas relações com a geomorfologia. Biota Neotropica, n. 10, v. 2, p. 31-38, 2010.

7. FEITOSA, M. C. B.; QUERINO, R. B.; HENRIQUES A. L. Perfil da fauna de vespas parasitóides (Insecta: Hymenoptera) em Reserva Florestal na Amazônia, Amazonas, Brasil. Entomotropica, n. 1, v. 22, p. 37-43, 2007.

8. FELFILI, J. M.; CARVALHO, F. A.; HAIDAR, R. F. Manual para o monitoramento de parcelas permanentes nos biomas Cerrado e Pantanal. Brasília: Universidade de Brasília, Departamento de Engenharia Florestal, 2005, 60 p.

9. FERREIRA, R. L.; MARQUES, M. G. S. M. A fauna de artrópodes de serrapilheira de áreas de monocultura cf. Eucalyptus sp. e Mata Secundária Heterogênea. Anais da Sociedade Entomológica do Brasil. n. 27, v. 3, p. 395-403, 1998.

10. JUNK, W. J.; BAYLEY, P. B.; SPARKS, R. E. The flood pulse concept in river-floodplain systems. 
In: DODGE, D. P. (ed.). Proceedings of the International Large River Symposium. Canadian Special Publication of Fisheries and Aquatic Science, Ottawa, p. 110-127, 1989.

11. KLINK, C. A.; MACHADO, R. B. A conservação do cerrado brasileiro. Megadiversidade, Brasília, n. 1 , v. $1,2005,155$ p.

12. MARQUES, M. I.; SOUZA, W. O.; SANTOS, G. B.; BATTIROLA, L. D.; ANJOS, K. C. Fauna de artrópodes de solo. In: FERNANDES, I. (org.). Biodiversidade no Pantanal de Poconé. Manaus: Instituto de Pesquisas Ecológicas, p. 73-102, 2010.

13. MARQUES, M. I.; ADIS, J.; BATTIROLA, Leandro D.; SANTOS, G. B.; CASTILHO, A. C. C. Arthropods associated witch a Forest of Attalea phalerata Mart. (Arecacear palm trees in the Northern Pantanal. In: JUNK, W. J. (org.) The Pantanal: Ecology, biodiversity and sustainable management of a large neotropica Iseasonal wetland. Pensoft: Bulgária, p. 431-468, 2011.

14. NUNES DA CUNHA, C.; JUNK, W. J. A preliminary classification of habitats of the Pantanal of Mato Grosso and Mato Grosso do Sul, and its relation to national and international wetland classification system. In: JUNK, W. J. (org.) The Pantanal: Ecology, biodiversity and sustainable management of a large neotropica Iseasonal wetland. Pensoft: Bulgária, p. 127141, 2011.

15. PRAXEDES, C.; MARTINS, M. B.; FURTADO, I.; AZEVEDO, M.; BASANTE, F. Estimativa da diversidade da fauna de serrapilheira em uma floresta densa de terra firme, Caxiuanã, Município de Melgaço - PA - Brasil. Estação Científica Ferreira Penna. Amazônia, p. 7-9, 2009.

16. RIBAS, C. R.; SCHOEREDER, J. H.; PIC, M.; SOARES, S. M. Tree heterogeneity, resource availability, and larger scale processes regulating ant species richness. Austral Ecology, n. 28, p. 305-314, 2003.

17. RIBEIRO, J. F.; WALTER, B. M. T. Fitofisionomias do bioma Cerrado: os biomas do Brasil. In: Cerrado: ambiente e flora. EMBRAPA: Planaltina, p.86-116, 1998.

18. SIGNOR, C. A.; FERNANDES, I. M.; PENHA, J. M. F. O Pantanal e o Sistema de Pesquisa. In: FERNANDES, I. (org.). Biodiversidade no Pantanal de Poconé. Manaus: Instituto de Pesquisas Ecológicas, 2010, $195 \mathrm{p}$.

19. VIANA, D. R.; ALVALÁ, R. C. dos Santos. Vegetation index performance for the Pantanal region during both dry and rainy seasons. Geografia, Rio Claro, v. 36, p. 143-158. 2011.

20. XIONG, S. J.; NILSSON, C. The effects of plant litter on vegetation: a meta-analysis. Journal of Ecology, n. 87, p. 984-94, 1999.

21. ZARDO, D. C.; CARNEIRO, A. P.; LIMA, L. G.; SANTOS-FILHO, M. Comunidade de artrópodes associada a serrapilheira de cerrado e mata de galeria, na Estação Ecológica Serra das Araras - Mato Grosso, Brasil. Uniara, n.2, v.13, p. 105-113, 2010. 